\title{
Eigenfrequencies and vibration modes of carbon nanotubes
}

\author{
Matteo Strozzi*, Leonid I. Manevitch ${ }^{\circ}$ \\ Valeri V. Smirnov ${ }^{\circ}$, Denis S. Shepelev ${ }^{\circ}$, Francesco Pellicano* \\ *Department of Engineering "Enzo Ferrari" \\ University of Modena and Reggio Emilia \\ Via Vignolese 905, 41125 Modena, Italy \\ ${ }^{\circ}$ N.N. Semenov Institute of Chemical Physics \\ Russian Academy of Sciences RAS \\ ul. Kosygina 4, 119991 Moscow, Russia
}

\begin{abstract}
The present work is concerned with the analysis of low-frequency linear vibrations of SWNTs: two approaches are presented: a fully analytical method based on a simplified theory and a semi-analytical method based on the theory of thin shells.

The semi-analytical approach (shortly called "numerical approach") is based on the Sanders-Koiter shell theory and the Rayleigh-Ritz numerical procedure. The nanotube deformation is described in terms of longitudinal, circumferential and radial displacement fields, which are expanded by means of a double mixed series based on Chebyshev polynomials. The Rayleigh-Ritz method is then applied to obtain numerically approximate natural frequencies and mode shapes.

The second approach is based on a reduced version of the Sanders-Koiter shell theory, obtained by assuming small ring and tangential shear deformations. These assumptions allow to condense both the longitudinal and the circumferential displacement fields. A fourth-order partial differential equation for the radial displacement field is derived. Eigenfunctions are formally obtained analytically, then the numerical solution of the dispersion equation gives the natural frequencies and the corresponding normal modes.

The methods are fully validated by comparing the natural frequencies of the SWNTs with data available in literature, namely: experiments, molecular dynamics simulations and finite element analyses. A comparison between the results of the numerical and analytical approach is carried out in order to check the accuracy of the last one.

It is worthwhile to stress that the analytical model allows to obtain results with very low computational effort. On the other hand the numerical approach is able to handle the most realistic boundary conditions of SWNTs (free-free, clamped-free) with extreme accuracy. Both methods are suitable for a forthcoming extension to multi-walled nanotubes and nonlinear vibrations.
\end{abstract}

Keywords: nanotubes, vibration, eigenvalues. 


\section{Introduction}

Carbon Nanotubes were discovered in 1991 by Iijima [1], who first analysed the synthesis of molecular carbon structures in the form of fullerenes and then reported the preparation of a new type of finite carbon structure consisting of needle-like tubes, the carbon nanotubes, described as helical microtubules of graphitic carbon.

Carbon Nanotubes (CNTs) are used as ultrahigh frequency nanomechanical resonators in a large number of nanoelectromechanical devices such as sensors, oscillators, charge detectors and field emission devices. The reduction of the size and the increment of the stiffness of a resonator magnify its resonant frequencies and reduce its energy consumption, improving its sensitivity.

The modal analysis of carbon nanotubes is important because it allows to obtain the resonant frequencies and mode shapes, which influence the mechanical and electronic properties of the nanotube resonators.

A large number of experiments and atomistic simulations were conducted both on single-walled (SWNTs) and multi-walled carbon nanotubes (MWNTs).

Rao et al. [2] studied the vibrations of SWNTs by using Raman scattering experimental techniques with laser excitation wavelengths in the range of the nanometers. They observed numerous Raman peaks, which correspond to vibrational modes of the nanotubes.

Bandow et al. [3] analysed the effect of the temperature growth on the diameter distribution and chirality of SWNTs by comparing different experimental techniques, such as electron microscopy, X-ray diffraction and Raman spectroscopy. They studied the effect of the catalysts on the tube yield and the evolution of the tube distribution vs. the environmental temperature.

Jorio et al. [4] studied the vibrations of SWNTs by resonant confocal micro-Raman spectroscopy. They developed a method to assign univocally the carbon nanotube chirality by measuring one radial breathing mode frequency and applying the theory of resonant transitions.

Because of their nanoscale size, it is very difficult to investigate the mechanical properties of the nanotubes using experimental techniques, which require the use of high resolution transmission electron microscopes and do not allow to separate easily the natural frequencies of the different vibration modes within the frequency spectrum. On the other hand, it was found that molecular dynamics simulations (MD) and finite element analyses (FE) provide good predictions of the mechanical behaviour of CNTs under external forces, with results close to the experiments [510].

Analytical and numerical methods have been recently developed in order to analyse classes of CNTs in a more general and efficient way; such methods are generally based on continuous models for the nanotube and allow a strong reduction of the number of degrees of freedom [11-14].

Eisenberger et al. [16] analysed the effect of the Van der Waals interactions on the vibration characteristics of multi-walled carbon nanotubes. An elastic multiple thin shell model was used. Based on the simplified Donnell shell equations, the natural frequencies and mode shapes of MWNTs with various radii and number of tubes were obtained. 
Wang et al. [15] examined the applicability and limitations of different simplified models of elastic cylindrical shells for general cases of static buckling and free vibrations of carbon nanotubes. They found that the simplified Flugge model, which retains the mathematical simplicity of the Donnell model, is in better agreement with the Flugge equations with respect to the Donnell theory.

In the present paper, we analyse the low-frequency linear vibrations of SWNTs by using two shell models. Applicability and limitations of these continuous models are investigated in detail.

The first approach is semi-analytical and it is based on the Rayleigh-Ritz numerical procedure, we call it shortly "numerical approach"; the Sanders-Koiter shell theory is considered in order to obtain the expressions of the elastic strain and kinetic energy. The nanotube deformation is described in terms of longitudinal, circumferential and radial displacement fields, which are expanded by means of a double mixed series based on Chebyshev polynomials for the longitudinal variable and harmonic functions for the circumferential variable. The Rayleigh-Ritz method is then applied to obtain numerically approximate natural frequencies and mode shapes.

The second approach consists of an analytical model based on a reduced version of the Sanders-Koiter shell theory, obtained by assuming small ring and tangential shear deformations. These assumptions allow to condense both the longitudinal and the circumferential displacement fields. A fourth-order partial differential equation for the radial displacement field is derived. Eigenfunctions are formally obtained analytically, then the numerical solution of the dispersion equation gives the natural frequencies and the corresponding normal modes.

In order to validate the present study, the natural frequencies of the carbon nanotube predicted by the present numerical model are compared with data available in literature: experiments, molecular dynamics simulations and finite element analyses. A comparison between the results of the numerical and analytical approach is carried out in order to check the accuracy of the last one. It is worthwhile to stress that the analytical model allows to obtain results with very low computational effort.

\section{Sanders-Koiter shell theory (numerical solution)}

First of all, it must be pointed out that in the present theory the size-effects are not considered; this simplification gives some limitations to the present model in terms of type of nanotube and dynamic conditions. The small scale effect has a significant influence on the dispersion of the flexural waves of SWNTs only for high frequencies [12]. Therefore, in order to make valid the assumptions of the present theory, the following two limitations have to be considered: the low part of the frequency spectrum can be analysed [15], carbon nanotubes with high aspect ratio and large diameter can be studied [17].

In Figure 1, a circular cylindrical shell having radius $R$, length $L$ and thickness $h$ is represented; a cylindrical coordinate system $(O ; x, \theta, z)$ is considered in order to take advantage from the axial symmetry of the structure, the origin $O$ of the reference system is located at the centre of one end of the cylindrical shell. In Figure 1, three displacement fields are represented: longitudinal $u(x, \theta, t)$, circumferential $v(x, \theta, t)$ and radial $w(x, \theta, t)$; the radial displacement field $w$ is considered positive outward 
and $(x, \theta)$ are the longitudinal and angular coordinates of an arbitrary point on the middle surface of the shell; $z$ is the radial coordinate along the thickness $h ; t$ is the time.
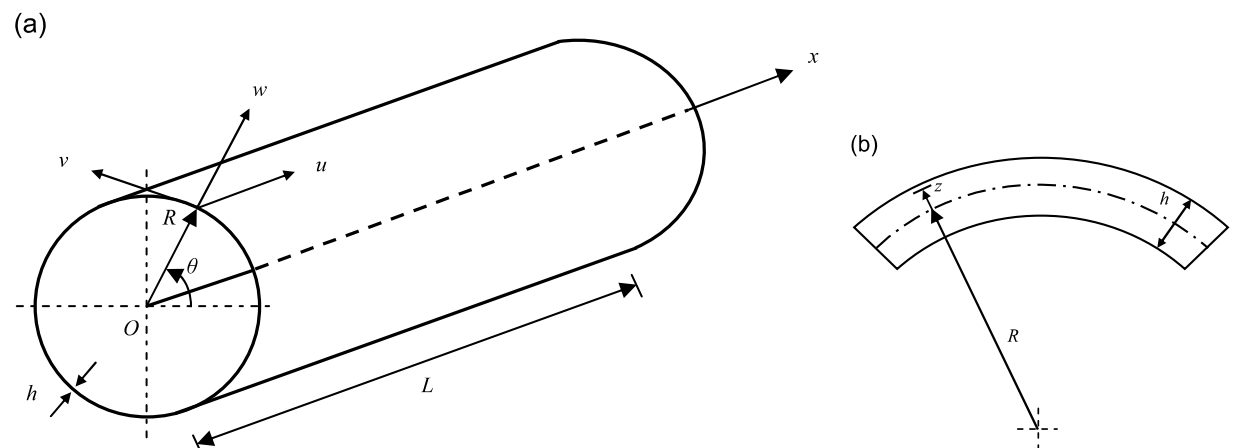

Figure 1. Geometry of the circular cylindrical shell. (a) Complete shell; (b) cross-section of the shell surface.

\subsection{Strain-displacement relationships}

It should be pointed out that, due to the nanoscale, CNTs present huge natural frequencies $(\mathrm{THz})$ and infinitesimal dimensions; this can induce numerical troubles to numerical algorithms if the governing equations are not transformed into a nondimensional form.

Here, the three displacement fields $(u, v, w)$ are nondimensionalized by means of the radius $R$ of the carbon nanotube

$$
u=R \tilde{u} \quad v=R \tilde{v} \quad w=R \tilde{w}
$$

where $(\tilde{u}, \tilde{v}, \tilde{w})$ are the nondimensional displacement fields.

In the Sanders-Koiter elastic thin shell theory, the transverse shear strains $\left(\gamma_{x z}, \gamma_{\theta z}\right)$ are neglected (Kirchhoff-Love's kinematic hypothesis). The tangential normal strains $\left(\varepsilon_{x}, \varepsilon_{\theta}\right)$ and the tangential shear strain $\gamma_{x \theta}$ at an arbitrary radius on the shell thickness are related to the middle surface strains $\left(\varepsilon_{x, 0}, \varepsilon_{\theta, 0}, \gamma_{x \theta, 0}\right)$ and to the changes in curvature and torsion of the middle surface of the shell $\left(k_{x}, k_{\theta}, k_{x \theta}\right)$ by the relationships [19]

$$
\varepsilon_{x}=\varepsilon_{x, 0}+z k_{x} \quad \varepsilon_{\theta}=\varepsilon_{\theta, 0}+z k_{\theta} \quad \gamma_{x \theta}=\gamma_{x \theta, 0}+z k_{x \theta}
$$

where $z$ is the distance of the arbitrary point on the thickness from the middle surface of the shell, according to the condition $(-h / 2 \leq z \leq h / 2)$, as shown in Figure 1.

The middle surface strains $\left(\varepsilon_{x, 0}, \varepsilon_{\theta, 0}, \gamma_{x \theta, 0}\right)$ are nondimensional parameters. The changes in curvature and torsion of the middle surface of the shell $\left(k_{x}, k_{\theta}, k_{x \theta}\right)$ are dimensional parameters, and they must be written in nondimensional form; this is achieved by considering the radius $R$. The middle surface strains and nondimensional changes in curvature and torsion can be written as follows [19]

$$
\begin{array}{ccc}
\tilde{\varepsilon}_{x, 0}=\alpha \frac{\partial \tilde{u}}{\partial \eta}=\varepsilon_{x, 0} & \tilde{\varepsilon}_{\theta, 0}=\frac{\partial \tilde{v}}{\partial \theta}+\tilde{w}=\varepsilon_{\theta, 0} & \tilde{\gamma}_{x \theta, 0}=\frac{\partial \tilde{u}}{\partial \theta}+\alpha \frac{\partial \tilde{v}}{\partial \eta}=\gamma_{x \theta, 0} \\
\tilde{k}_{x}=-\alpha^{2} \frac{\partial^{2} \tilde{w}}{\partial \eta^{2}}=R k_{x} & \tilde{k}_{\theta}=\frac{\partial \tilde{v}}{\partial \theta}-\frac{\partial^{2} \tilde{w}}{\partial \theta^{2}}=R k_{\theta} & \tilde{k}_{x \theta}=-2 \alpha \frac{\partial^{2} \tilde{w}}{\partial \eta \partial \theta}+\frac{3}{2} \alpha \frac{\partial \tilde{v}}{\partial \eta}-\frac{1}{2} \frac{\partial \tilde{u}}{\partial \theta}=R k_{x \theta}
\end{array}
$$


where $\left(\tilde{\varepsilon}_{x, 0}, \tilde{\varepsilon}_{\theta, 0}, \tilde{\gamma}_{x \theta, 0}\right)=\left(\varepsilon_{x, 0}, \varepsilon_{\theta, 0}, \gamma_{x \theta, 0}\right)$ are middle surface strains of the shell, $\left(\tilde{k}_{x}, \tilde{k}_{\theta}, \tilde{k}_{x \theta}\right)$ are the nondimensional middle surface changes in curvature and torsion, $\eta=x / L$ is the nondimensional longitudinal coordinate of the shell and $\alpha=R / L$.

\subsection{Force and moment resultants}

The nondimensional force $\left(\tilde{N}_{x}, \tilde{N}_{\theta}, \tilde{N}_{x \theta}, \tilde{Q}_{x}, \tilde{Q}_{\theta}\right)$ and moment $\left(\tilde{M}_{x}, \tilde{M}_{\theta}, \tilde{M}_{x \theta}\right)$ resultants can be written in the following form

$$
\begin{array}{ccc}
\tilde{N}_{x}=\tilde{\varepsilon}_{x, 0}+v \tilde{\varepsilon}_{\theta, 0}=\frac{N_{x}}{J} & \tilde{N}_{\theta}=\tilde{\varepsilon}_{\theta, 0}+v \tilde{\varepsilon}_{x, 0}=\frac{N_{\theta}}{J} & \tilde{N}_{x \theta}=\frac{(1-v)}{2} \tilde{\gamma}_{x \theta, 0}=\frac{N_{x \theta}}{J} \\
\tilde{Q}_{x}=R\left[\tilde{k}_{x, x}+v \tilde{k}_{\theta, x}+\frac{(1-v)}{2 R} \tilde{k}_{\theta x, \theta}\right]=\frac{R^{2}}{D} Q_{x} & \tilde{Q}_{\theta}=R\left[\frac{(1-v)}{2} \tilde{k}_{x \theta, x}+\frac{1}{R}\left(\tilde{k}_{\theta, \theta}+v \tilde{k}_{x, \theta}\right)\right]=\frac{R^{2}}{D} Q_{\theta} \\
\tilde{M}_{x}=\tilde{k}_{x}+v \tilde{k}_{\theta}=\frac{R}{D} M_{x} & \tilde{M}_{\theta}=\tilde{k}_{\theta}+v \tilde{k}_{x}=\frac{R}{D} M_{\theta} & \tilde{M}_{x \theta}=\frac{(1-v)}{2} \tilde{k}_{x \theta}=\frac{R}{D} M_{x \theta}
\end{array}
$$

where $\left(N_{x}, N_{\theta}, N_{x \theta}, Q_{x}, Q_{\theta}\right)$ are the force resultants per unit length of the shell, $\left(M_{x}, M_{\theta}, M_{x \theta}\right)$ are the moment resultants per unit length of the shell, $J=E h /\left(1-v^{2}\right)$ and $D=E h^{3} /\left(12\left(1-v^{2}\right)\right)$.

\subsection{Elastic strain energy}

According to the Sanders-Koiter theory, the elastic strain energy $U$ of a circular cylindrical shell is written in the form [21]

$$
U=\frac{1}{2} L R \int_{0}^{1} \int_{0}^{2 \pi} \int_{-h / 2}^{h / 2}\left(\sigma_{x} \varepsilon_{x}+\sigma_{\theta} \varepsilon_{\theta}+\tau_{x \theta} \gamma_{x \theta}\right) d \eta d \theta d z
$$

The elastic strain energy $U$ can be nondimensionalized, let $\tilde{U}$ be the nondimensional elastic strain energy, it can be expressed in the form

$$
\tilde{U}=\frac{1}{2} \frac{1}{1-v^{2}}\left[\int_{0}^{1} \int_{0}^{2 \pi}\left(\tilde{\varepsilon}_{x, 0}^{2}+\tilde{\varepsilon}_{\theta, 0}^{2}+2 v \tilde{\varepsilon}_{x, 0} \tilde{\varepsilon}_{\theta, 0}+\frac{(1-v)}{2} \tilde{\gamma}_{x \theta, 0}^{2}\right) d \eta d \theta+\frac{\beta^{2}}{12} \int_{0}^{12 \pi} \int_{0}^{2 \pi}\left(\tilde{k}_{x}^{2}+\tilde{k}_{\theta}^{2}+2 v \tilde{k}_{x} \tilde{k}_{\theta}+\frac{(1-v)}{2} \tilde{k}_{x \theta}^{2}\right) d \eta d \theta\right]=\frac{U}{A}
$$

where $A=E h L R$ and $\beta=h / R$.

\subsection{Kinetic energy}

The kinetic energy $T$ of a cylindrical shell (rotary inertia effect being neglected) is given by [21]

$$
T=\frac{1}{2} \rho h L R \int_{0}^{1} \int_{0}^{2 \pi}\left(\dot{u}^{2}+\dot{v}^{2}+\dot{w}^{2}\right) d \eta d \theta
$$

where $\rho$ is the mass density of the shell and the overdot denotes a time derivative.

The time variable $t$ can be nondimensionalized by introducing a reference natural frequency $\omega_{0}$ in the following form [20]

$$
t=\omega_{0}^{-1} \tau \quad \omega_{0}=\sqrt{\frac{E}{\left(1-v^{2}\right) \rho R^{2}}}
$$


where $\tau$ is the nondimensional time variable.

The velocity fields $(\dot{u}, \dot{v}, \dot{w})$ can be nondimensionalized by considering the radius $R$ and the reference natural frequency $\omega_{0}$ in the following form

$$
\dot{u}=R \omega_{0} \tilde{u}^{\prime} \tilde{u}^{\prime}=\frac{d \tilde{u}}{d \tau} \dot{v}=R \omega_{0} \tilde{v}^{\prime} \tilde{v}^{\prime}=\frac{d \tilde{v}}{d \tau} \dot{w}=R \omega_{0} \tilde{w}^{\prime} \tilde{w}^{\prime}=\frac{d \tilde{w}}{d \tau}
$$

where $\left(\tilde{u}^{\prime}, \tilde{v}^{\prime}, \tilde{w}^{\prime}\right)$ are the nondimensional velocity fields.

Let $\tilde{T}$ be the nondimensional kinetic energy, which is expressed in the form

$$
\tilde{T}=\frac{1}{2} \int_{0}^{1} \int_{0}^{2 \pi}\left(\tilde{u}^{2}+\tilde{v}^{2}+\tilde{w}^{2}\right) d \eta d \theta=\frac{1}{2} \int_{0}^{1} \int_{0}^{2 \pi}\left[\left(\frac{d \tilde{u}}{d \tau}\right)^{2}+\left(\frac{d \tilde{v}}{d \tau}\right)^{2}+\left(\frac{d \tilde{w}}{d \tau}\right)^{2}\right] d \eta d \theta=\frac{T}{\gamma A}
$$

where $\gamma=\rho R^{2} \omega_{0}^{2} / E$.

\subsection{Linear vibration analysis (complete Sanders-Koiter shell theory)}

In the linear vibration analysis, the three nondimensional displacement fields are expanded by using a double mixed series, then the Rayleigh-Ritz method is applied to the linearized formulation of the problem, in order to obtain approximated eigenfunctions. The linear vibration analysis is carried out considering only the quadratic terms in equation (8).

A modal vibration, i.e., a synchronous motion, can be formally written in the form [43]

$$
\tilde{u}(\eta, \theta, \tau)=\tilde{U}(\eta, \theta) f(\tau) \quad \tilde{v}(\eta, \theta, \tau)=\tilde{V}(\eta, \theta) f(\tau) \quad \tilde{w}(\eta, \theta, \tau)=\tilde{W}(\eta, \theta) f(\tau)
$$

where $\tilde{U}(\eta, \theta), \tilde{V}(\eta, \theta), \tilde{W}(\eta, \theta)$ describe the mode shape of the shell and $f(\tau)$ represents the common time law, which is supposed to be the same for each displacement field in the modal vibration analysis.

The mode shape $(\tilde{U}, \tilde{V}, \tilde{W})$ is expanded by means of a double mixed series, in terms of $m$-th order Chebyshev polynomials $T_{m}{ }^{*}(\eta)$ in the axial direction and harmonic functions $(\cos n \theta, \sin n \theta)$ in the circumferential direction, in the following form [23]

$$
\tilde{U}(\eta, \theta)=\sum_{m=0}^{M_{n}} \sum_{n=0}^{N} \tilde{U}_{m, n} T_{m}^{*}(\eta) \cos n \theta \tilde{V}(\eta, \theta)=\sum_{m=0}^{M_{v}} \sum_{n=0}^{N} \tilde{V}_{m, n} T_{m}^{*}(\eta) \sin n \theta \tilde{W}(\eta, \theta)=\sum_{m=0}^{M_{v}} \sum_{n=0}^{N} \tilde{W}_{m, n} T_{m}^{*}(\eta) \cos n \theta
$$

where $T_{m}{ }^{*}=T_{m}(2 \eta-1), m$ is the polynomials degree, $n$ denotes the number of nodal diameters and $\left(\tilde{U}_{m, n}, \tilde{V}_{m, n}, \tilde{W}_{m, n}\right)$ are unknown coefficients.

\subsubsection{Boundary conditions}

Clamped and free SWNTs are now analysed; the boundary conditions are imposed by applying constraints to the unknown coefficients $\left(\tilde{U}_{m, n}, \tilde{V}_{m, n}, \tilde{W}_{m, n}\right)$ of the expansions (15).

\subsubsection{Clamped-clamped}

Clamped - clamped boundary conditions are given by [23]

$$
\tilde{u}=0 \quad \tilde{v}=0 \quad \tilde{w}=0 \quad \tilde{w}_{, \eta}=0 \quad \eta=0,1
$$


where $(\cdot)_{, \eta}=\partial(\cdot) / \partial \eta$.

The previous conditions imply the following equations [23]

$\sum_{m=0}^{M_{u}} \tilde{U}_{m, n} T_{m}^{*}(\eta)=0 \sum_{m=0}^{M_{v}} \tilde{V}_{m, n} T_{m}^{*}(\eta)=0 \sum_{m=0}^{M_{m}} \tilde{W}_{m, n} T_{m, \eta}^{*}(\eta)=0 \sum_{m=0}^{M_{w}} \tilde{W}_{m, n} T_{m}^{*}(\eta)=0 \quad \theta \in[0,2 \pi] \quad n \in[0, N]$

The linear algebraic system given by the equations (17) can be solved analytically in terms of the coefficients $\left(\tilde{U}_{0, n}, \tilde{U}_{1, n}, \tilde{V}_{0, n}, \tilde{V}_{1, n}, \tilde{W}_{0, n}, \tilde{W}_{1, n}, \tilde{W}_{2, n}, \tilde{W}_{3, n}\right)$, for $\mathrm{n} \in[0, \mathrm{~N}]$.

\subsubsection{Free-free}

Free - free boundary conditions are given by [23]

$$
\tilde{N}_{x}=0 \quad \tilde{N}_{x \theta}+\tilde{M}_{x \theta}=0 \quad \tilde{Q}_{x}+\frac{\partial \tilde{M}_{x \theta}}{\partial \theta}=0 \quad \tilde{M}_{x}=0 \quad \eta=0,1
$$

It can be observed that the boundary conditions (18) applied at the free edges of the SWNTs are of natural type; since the Rayleigh-Ritz method is used for finding the solution, just the geometric boundary conditions have to be exactly satisfied: it means that it is not necessary to satisfy the natural conditions (18) by the expansions (15) since they will be satisfied by the minimization of the energy of the system.

\subsubsection{Rayleigh-Ritz method}

The maximum number of variables needed for describing a general vibration mode with $n$ nodal diameters is obtained by the relation $\left(N_{p}=M_{u}+M_{v}+M_{w}+3-p\right)$, where $\left(M_{u}=M_{v}=M_{w}\right)$ denote the degree of the Chebyshev polynomials and $p$ describes the number of equations needed to satisfy the boundary conditions.

A specific convergence analysis is carried out to select the degree of the Chebyshev polynomials: degree 11 is found suitably accurate, $\left(M_{u}=M_{v}=M_{w}=11\right)$, see Refs. [21-23] for the details.

For a multi-mode analysis including different values of nodal diameters $n$, the number of degrees of freedom of the system is computed by the relation $\left(N_{\max }=N_{p}\right.$ $\times(N+1))$, where $N$ represents the maximum value of the nodal diameters $n$ considered.

For example, in the case of a SWNT with free edges $(p=0)$, the number of degrees of freedom of the system with $(n=2)$ nodal diameters is equal to $\left(N_{\max }=N_{p} \times(N+\right.$ $1)=36 \times(2+1)=108)$.

Equations (14) are inserted into the expressions of the elastic strain energy $\tilde{U}$, eq. (9), and kinetic energy $\tilde{T}$, eq. (13), to compute the Rayleigh quotient $R(\tilde{\mathrm{q}})=\tilde{U}_{\max } /$ $\tilde{T}^{*}$, where $\tilde{U}_{\max }=\max (\tilde{U})$ is the maximum of the potential energy during a modal vibration, $\tilde{T}^{*}=\tilde{T}_{\max } / \omega^{2}, \tilde{T}_{\max }=\max (\tilde{T})$ is the maximum of the kinetic energy during a modal vibration, $\omega$ represents the circular frequency of the synchronous harmonic motion $f(\tau)=\cos \omega \tau$; $\tilde{\mathrm{q}}$ is a vector containing all the unknown variables (its structure depends on the boundary conditions) [23]

$$
\tilde{\mathrm{q}}=\left[\begin{array}{lllll}
\ldots & \tilde{U}_{m, m} & \tilde{V}_{m, n} & \tilde{W}_{m, n} & \cdots
\end{array}\right]^{T}
$$


After imposing the stationarity to the Rayleigh quotient, one obtains the eigenvalue problem [23]

$$
\left(-\omega^{2} \tilde{\mathrm{M}}+\tilde{\mathrm{K}}\right) \tilde{\mathrm{q}}=\tilde{0}
$$

which furnishes approximate natural frequencies (eigenvalues) and mode shapes (eigenvectors and eigenfunctions).

The approximate mode shape of the $j$-th mode is given by the equations (15), where the coefficients $\left(\tilde{U}_{m, n}, \tilde{V}_{m, n}, \tilde{W}_{m, n}\right)$ are substituted with $\left(\tilde{U}_{m, n}^{(j)}, \tilde{V}_{m, n}^{(j)}, \tilde{W}_{m, n}^{(j)}\right)$, which denote the components of the $j$-th eigenvector $\tilde{\mathrm{q}}_{j}$ of the equation (20). The vector function [23]

$$
\tilde{\mathrm{Q}}^{(j)}(\eta, \theta)=\left[\begin{array}{ccc}
\tilde{U}^{(j)}(\eta, \theta) & \tilde{V}^{(j)}(\eta, \theta) & \tilde{W}^{(j)}(\eta, \theta)
\end{array}\right]^{T}
$$

is the approximation of the $j$-th eigenfunction vector of the original problem.

\section{Reduced Sanders-Koiter shell theory (analytical solution)}

In the present section, a reduced form of the Sanders-Koiter shell theory is developed. Also in this model, the transverse shear strains $\left(\gamma_{x z}, \gamma_{\theta z}\right)$ are neglected. In the present work, we consider small amplitude vibrations of CNTs, and a potential energy is predominantly due to bending, torsion and longitudinal tensions. Therefore, we can suppose that both in-plane circumferential normal strain and tangential shear strain of the middle surface of the shell $\left(\varepsilon_{\theta, 0}, \gamma_{x \theta, 0}\right)$ are "small differences of relatively large quantities" [20], and they can be neglected. Due to this assumption, one can reduce the number of dependent variables; both the longitudinal and the circumferential displacements can be expressed via the radial one.

By assuming the linear expansions of the longitudinal $\tilde{u}$, circumferential $\tilde{v}$ and radial $\tilde{w}$ displacement fields as

$$
\tilde{u}(\eta, \theta, \tau)=\hat{U}(\eta) \cos (n \theta) \mathrm{e}^{-\mathrm{i} \omega \tau} \tilde{v}(\eta, \theta, \tau)=\hat{V}(\eta) \sin (n \theta) \mathrm{e}^{-\mathrm{i} \omega \tau} \tilde{w}(\eta, \theta, \tau)=\hat{W}(\eta) \cos (n \theta) \mathrm{e}^{-\mathrm{i} \omega \tau}
$$

using the condition of absence of ring (in-plane circumferential) deformation effects

$$
\tilde{\varepsilon}_{\theta, 0}=\frac{\partial \tilde{v}}{\partial \theta}+\tilde{w}=0
$$

and the condition of absence of tangential shear deformation effects

$$
\tilde{\gamma}_{x \theta, 0}=\frac{\partial \tilde{u}}{\partial \theta}-\alpha \frac{\partial \tilde{v}}{\partial \eta}=0
$$

we can obtain the following expressions for the nondimensional variables $\hat{V}$ and $\hat{U}$ as functions of the radial displacement $\hat{W}$

\subsection{Equations of motion}

$$
\hat{V}(\eta)=-\frac{\hat{W}(\eta)}{n} \quad \hat{U}(\eta)=-\frac{\alpha}{n^{2}} \frac{\partial \hat{W}(\eta)}{\partial \eta}
$$

In order to get the adequate equation of motion in terms of $\hat{W}$ one cannot insert these relations into equations of motion (1.120) of Ref. [20] because of the "reactive" nature of the circumferential and tangential shear reduced forces. Therefore, we have to start from the following "force" form of the equations of motion

$$
\frac{\partial^{2} \tilde{u}}{\partial \tau^{2}}-\alpha \frac{\partial \tilde{N}_{x}}{\partial \eta}-\frac{\partial \tilde{N}_{x \theta}}{\partial \theta}+\frac{\beta}{2} \frac{\partial \tilde{M}_{x \theta}}{\partial \theta}=0
$$




$$
\begin{aligned}
& \frac{\partial^{2} \tilde{v}}{\partial \tau^{2}}-\frac{\partial \tilde{N}_{\theta}}{\partial \theta}-\alpha \frac{\partial \tilde{N}_{x \theta}}{\partial \eta}-\beta \frac{\partial^{2} \tilde{M}_{\theta}}{\partial \theta^{2}}-\frac{3}{2} \alpha \beta \frac{\partial \tilde{M}_{x \theta}}{\partial \eta}=0 \\
& \frac{\partial^{2} \tilde{w}}{\partial \tau^{2}}+\tilde{N}_{\theta}-\alpha^{2} \beta \frac{\partial^{2} \tilde{M}_{x}}{\partial \eta^{2}}-\beta \frac{\partial^{2} \tilde{M}_{\theta}}{\partial \theta^{2}}-2 \alpha \beta \frac{\partial^{2} \tilde{M}_{x \theta}}{\partial \eta \partial \theta}=0
\end{aligned}
$$

where $\left(\tilde{N}_{x}, \tilde{N}_{\theta}, \tilde{N}_{x \theta}\right)$ and $\left(\tilde{M}_{x}, \tilde{M}_{\theta}, \tilde{M}_{x \theta}\right)$ are the reduced force and moment resultants, respectively.

The procedure of the equations reduction consists of a consecutive definition of the expressions for $\tilde{N}_{x \theta}$ and $\tilde{N}_{\theta}$ from the equations (26) and (27), and their substitution into the equation (28).

Let us consider these actions in detail.

We take into account that the reduced forces and momenta have the following form

$$
\begin{gathered}
\tilde{N}_{x}(\eta, \theta, \tau)=\varphi_{\eta}(\eta) \cos (n \theta) \mathrm{e}^{-\mathrm{i} \omega \tau} \tilde{N}_{\theta}(\eta, \theta, \tau)=\varphi_{\theta}(\eta) \cos (n \theta) \mathrm{e}^{-\mathrm{i} \omega \tau} \\
\tilde{N}_{x \theta}(\eta, \theta, \tau)=\varphi_{\eta \theta}(\eta) \sin (n \theta) \mathrm{e}^{-\mathrm{i} \omega \tau} \tilde{M}_{x}(\eta, \theta, \tau)=\mu_{\eta}(\eta) \cos (n \theta) \mathrm{e}^{-\mathrm{i} \omega \tau} \\
\tilde{M}_{\theta}(\eta, \theta, \tau)=\mu_{\theta}(\eta) \cos (n \theta) \mathrm{e}^{-\mathrm{i} \omega \tau} \tilde{M}_{x \theta}(\eta, \theta, \tau)=\mu_{\eta \theta}(\eta) \sin (n \theta) \mathrm{e}^{-\mathrm{i} \omega \tau}
\end{gathered}
$$

Substituting these relationships into equations (26-28), we obtain the relations

$$
\begin{gathered}
-\omega^{2} \hat{U}-\alpha \frac{\partial \varphi_{\eta}}{\partial \eta}-n \varphi_{\eta \theta}+n \frac{\beta}{2} \mu_{\eta \theta}=0 \\
{ }^{2} \hat{V}+n \quad+n^{2} \quad \frac{3}{2}-=0 \\
-\omega^{2} \hat{W}+\varphi_{\theta}-\alpha^{2} \beta \frac{\partial^{2} \mu_{\eta}}{\partial \eta^{2}}+\beta n^{2} \mu_{\theta}-2 \alpha \beta n \frac{\partial \mu_{\eta \theta}}{\partial \eta}=0
\end{gathered}
$$

Then, we can obtain $\varphi_{\eta \theta}$ from equation (32) and substitute the result into equation (33), the same procedure applies to $\varphi_{\theta}$ that is substituted into equation (34).

The last step is to express the remaining reduced forces and momenta via the corresponding deformations excluding the variables $\hat{V}$ and $\hat{U}$ by the relations (25). The final equation of motion for the nondimensional radial displacement field $\hat{W}$ is given by

$$
\begin{gathered}
\frac{\partial^{2} \hat{W}}{\partial \tau^{2}}+\frac{n^{2}\left(n^{2}-1\right)^{2}}{n^{2}+1} \beta^{2} \hat{W}-\frac{\left(n^{2}-1\right)\left(n^{2}-1+v\right)}{6\left(n^{2}+1\right)} \alpha^{2} \beta^{2} \frac{\partial^{2} \hat{W}}{\partial \eta^{2}} \\
-\frac{\alpha^{2}}{n^{2}\left(n^{2}+1\right)} \frac{\partial^{4} \hat{W}}{\partial \tau^{2} \partial \eta^{2}}+\frac{12+n^{4} \beta^{2}}{12 n^{2}\left(n^{2}+1\right)} \alpha^{4} \frac{\partial^{4} \hat{W}}{\partial \eta^{4}}=0
\end{gathered}
$$

where the time dependence of the radial displacement is taken into account explicitly.

The same equation can be obtained taking into account only the bending, torsion and longitudinal stresses in the expression of the strain energy and expressing the longitudinal and circumferential displacements via the radial one before the application of the variational procedure.

\subsection{Boundary conditions}

In the present section, both periodic and free-free boundary conditions are considered. 


\subsubsection{Periodic boundary conditions}

In the case of periodic boundary conditions, equation (35) leads to the dispersion equation

$$
\omega^{2}=\frac{\beta^{2} n^{4}\left(n^{2}-1\right)^{2}+2 \alpha^{2} \beta^{2} \pi^{2} j^{2} n^{2}\left(n^{2}-1\right)\left(n^{2}-(1-v)\right)+\alpha^{4}\left(12+\beta^{2} n^{4}\right) \pi^{4} j^{4}}{12\left(n^{4}+n^{2}+\alpha^{2} \pi^{2} j^{2}\right)}
$$

where $j$ describes the number of half-waves along the CNT axis.

\subsubsection{Free-free boundary conditions}

Using equation (35) and assuming $\hat{W}=\bar{W} e^{i \omega \tau}$ one obtains

$$
-\omega^{2} \bar{W}+\Omega^{2} \bar{W}-c^{2} \frac{\partial^{2} \bar{W}}{\partial \eta^{2}}+\omega^{2} A \frac{\partial^{2} \bar{W}}{\partial \eta^{2}}+B \frac{\partial^{4} \bar{W}}{\partial \eta^{4}}=0
$$

where

$$
\Omega^{2}=\frac{n^{2}\left(n^{2}-1\right)^{2}}{12\left(n^{2}+1\right)} \beta^{2} \quad c^{2}=\frac{\left(n^{2}-1\right)\left(n^{2}-1+v\right)}{6\left(n^{2}+1\right)} \alpha^{2} \beta^{2} \quad A=\frac{\alpha^{2}}{n^{2}\left(n^{2}+1\right)} \quad B=\frac{12+n^{4} \beta^{2}}{12 n^{2}\left(n^{2}+1\right)} \alpha^{4}
$$

We can factorize equation (37) as follows

$$
B\left(\frac{\partial^{2}}{\partial \eta^{2}}+\mu^{2}\right)\left(\frac{\partial^{2}}{\partial \eta^{2}}-\gamma^{2}\right) \bar{W}=0
$$

where the parameters $\mu$ and $\gamma$ are coupled by the relations

$$
\mu^{2} \gamma^{2}=\frac{\omega^{2}-\Omega^{2}}{B} \quad \mu^{2}-\gamma^{2}=\frac{\omega^{2} A-c^{2}}{B}
$$

The general solution of equation (39) contains both a harmonic-type solution and an aperiodic exponential-type one in $\eta$; the latter one plays the role of the edge layer, where the parameter $\gamma$ specifies its magnitude. In such a case the parameter $\mu$ is an effective wave number.

This equation should be completed with the free-free boundary conditions at $(\eta=0)$ and $(\eta=1)$, in terms of the radial displacement $\bar{W}$.

The first boundary condition corresponds to the absence of bending moment $\tilde{M}_{x}$ at the free edges of the carbon nanotube, which leads to the following relation

$$
\tilde{M}_{x}=0 \Rightarrow \alpha^{2} \frac{\partial^{2} \bar{W}}{d \eta^{2}}-v\left(n^{2}-1\right) \bar{W}=0 \quad \eta=(0,1)
$$

The second boundary condition is related with the transverse force $\tilde{Q}_{x}$ combined with the torsional moment $\tilde{M}_{x \theta}$ at the free edges of the carbon nanotube, and it leads to the following relation

$$
\tilde{Q}_{x}+\frac{1}{R} \frac{\partial \tilde{M}_{x \theta}}{\partial \theta}=0 \Rightarrow \alpha^{2} \frac{\partial^{3} \bar{W}}{\partial \eta^{3}}-\left(n^{2}-1\right)(2-v) \frac{\partial \bar{W}}{\partial \eta}=0 \quad \eta=(0,1)
$$

It should be noted that the two remaining boundary conditions are

$$
\tilde{N}_{x}=0 \quad \tilde{N}_{x \theta}+\tilde{M}_{x \theta}=0
$$

such conditions are satisfied thanks to the exclusion procedure discussed above.

\subsection{General solution}

The general solution of the equation (39) can be written as follows

$$
\bar{W}(\eta)=c_{1} \cos \left(\mu \eta+\delta_{1}\right)+c_{2} \exp \left(-\gamma \eta+\delta_{2}\right)+c_{3} \exp \left(\gamma \eta+\delta_{3}\right)
$$

where $\left(c_{1}, c_{2}, c_{3}\right)$ and $\left(\delta_{1}, \delta_{2}, \delta_{3}\right)$ are nondimensional parameters to be determined. 
In equation (44), there are both symmetric and asymmetric constituents corresponding to an even and odd number of half-waves along the $\eta$-axis of the CNT.

The respective values of the nondimensional parameters for the symmetric solution (even number of longitudinal half-waves) are

$$
c_{2}=c_{3} \quad \delta_{1}=-\frac{\mu}{2} \quad \delta_{2}=\delta_{3}=-\frac{\gamma}{2}
$$

and in the asymmetric one (odd number of longitudinal half-waves) we have

$$
c_{2}=-c_{3} \quad \delta_{1}=-\frac{\mu}{2}-\frac{\pi}{2} \quad \delta_{2}=\delta_{3}=-\frac{\gamma}{2}
$$

We can rewrite the symmetric and asymmetric solutions as follows

$$
\begin{gathered}
\bar{W}_{s}=\cos [\mu(\eta-1 / 2)]+b \sinh ^{2}\left[\frac{\gamma}{2}(\eta-1 / 2)\right] \\
\bar{W}_{a}=\sin [\mu(\eta-1 / 2)]+b \sinh [\gamma(\eta-1 / 2)]
\end{gathered}
$$

where the parameter $b$ plays the role of the edge layer amplitude.

By substituting these expressions into relations (41) and (42), we finally obtain two transcendent equations with respect to parameters $\mu$ and $b$, which must be solved numerically.

\section{Numerical results}

In Table 1, effective and equivalent parameters of Single-Walled Carbon Nanotubes are reported [18]. These parameters are used to carry out comparisons with experiments and MD simulations, i.e. the model validation.

The diameter $D$ of a CNT can be directly calculated from its chirality indices $(r, s)$ as follows

$$
D=\frac{a}{\pi} \sqrt{\left(r^{2}+r s+s^{2}\right)}
$$

$r$ and $s$ identify the chiral vector, which gives the rolling direction of a honeycomb crystal lattice of graphene; $a=0.246 \mathrm{~nm}$.

Therefore, a carbon nanotube modelled as a thin circular cylindrical shell is uniquely described by the length $L$ and by the indices $(r, s)$, which allow to determine the CNT diameter $D$. $L$ and $D$ are mutually connected by the aspect ratio $\chi=L / D$. Therefore, $(r, s, \chi)$ are the independent variables of a SWNT.

\begin{tabular}{|c|c|}
\hline Effective thickness $h_{0}(\mathrm{~nm})$ & $0.10 \div 0.15$ \\
\hline Equivalent thickness $h(\mathrm{~nm})$ & 0.066 \\
\hline Effective Young's modulus $E_{0}(\mathrm{TPa})$ & $1.0 \div 2.0$ \\
\hline Equivalent Young's modulus $E(\mathrm{TPa})$ & 5.5 \\
\hline Effective Poisson's ratio $v_{0}$ & $0.12 \div 0.28$ \\
\hline Equivalent Poisson's ratio $v$ & 0.19 \\
\hline Surface density of graphite $\sigma\left(\mathrm{kg} / \mathrm{m}^{2}\right)$ & $7.718 \times 10^{-7}$ \\
\hline Equivalent mass density $\rho\left(\mathrm{kg} / \mathrm{m}^{3}\right)$ & 11700 \\
\hline
\end{tabular}

Table 1. Effective and equivalent parameters of the Single-Walled Carbon Nanotubes [18]. 


\subsection{Validation of the numerical approach in linear field}

In this section, the numerical model based on the Sanders-Koiter shell theory is validated in linear field. The natural frequencies of the carbon nanotubes based on this theory are compared with data available in the literature: experiments, Molecular Dynamics (MD) simulations. In Tables 2-3 all the comparisons are reported. These comparisons show that the Sanders-Koiter theory and the present approach of solution give excellent results in terms of natural frequencies. Furthermore, this proves that the equivalent parameters are correct. In the following subsections, detailed comments regarding this validation are given.

\subsubsection{Radial Breathing Mode (RBM)}

The RBM is the specific vibrational mode which is often used in order to identify experimentally the CNTs by Resonant Raman Spectroscopy.

This mode corresponds to the "vibrational" numbers $(j=0, n=0)$, and its natural frequency can be easily calculated in the framework of the Sanders-Koiter elastic shell theory. The RBM appears only in the case of free-free boundary conditions.

The existing data for vibrations of CNTs are mainly focused on the radial breathing mode of SWNTs and MWNTs because the spectrum of nanotubes is quite complex. Moreover, the natural frequency associated with the radial breathing mode of SWNTs is inversely proportional to the tube diameter and independent from the length (aspect ratio $\chi$ ) and chirality (symmetry) of the nanotube.

For the radial breathing mode, the radial displacement field $\tilde{w}(\eta, \theta, \tau)$ is spatially uniform, i.e., it is independent of $\eta$ and $\theta(\tilde{w}=\tilde{w}(\tau))$. In this special case, the bending stiffness of the SWNTs does not appear because the radial breathing vibration does not involve the bending deformation and corresponds to an uniaxial stress state of the graphene sheet.

Resonant Raman Spectroscopy (RRS) provides a powerful technique to study the quantum properties of electrons and phonons in carbon nanotubes and to determine their atomic structure, i.e., the chirality indices $(r, s)$, of an isolated SWNT. The radial breathing mode exhibits strong resonant characteristics in the Raman spectra, because it corresponds to the symmetric in-phase motion of all the carbon atoms in the radial direction of the tube. In this configuration all the carbon atoms undergo the same radial displacement.

The RRS of a SWNT allows a unique assignment of its chirality to be made by measuring the RBM frequency $\omega_{R B M}$ and using the theory of the resonant transitions. By considering the frequency and the intensity of the RBM mode in the RRS spectra, it is possible to assign the correct chirality $(r, s)$ to the resonant SWNT.

The natural frequencies of the radial breathing vibration mode based on the present numerical model are in good agreement with the experimental RRS results. Table 2 presents a comparison for different armchair, zigzag and chiral SWNTs under freefree boundary conditions, relative errors are less than 5\%, it appears a satisfactory accuracy. 


\begin{tabular}{|c|c|c|c|}
\hline \multicolumn{3}{|c|}{ Natural frequency (THz) } & Difference \% \\
\hline$(r, s)$ & CSKT - Present model & RRS - Ref. [4] & \\
\hline$(8,7)$ & 6.905 & 7.165 & 3.63 \\
\hline$(10,5)$ & 6.785 & 7.105 & 4.50 \\
\hline$(11,4)$ & 6.669 & 6.865 & 2.85 \\
\hline$(14,1)$ & 6.177 & 6.295 & 1.87 \\
\hline$(18,0)$ & 5.025 & 5.276 & 4.76 \\
\hline$(17,2)$ & 4.964 & 5.216 & 4.83 \\
\hline$(16,4)$ & 4.895 & 5.066 & 3.37 \\
\hline$(15,6)$ & 4.788 & 4.947 & 3.21 \\
\hline$(11,11)$ & 4.711 & 4.917 & 4.19 \\
\hline$(19,1)$ & 4.594 & 4.797 & 4.23 \\
\hline
\end{tabular}

Table 2. Natural frequencies of the radial breathing mode $(j=0, n=0)$ : comparisons between the complete Sanders-Koiter theory (CSKT) and the Resonant Raman Spectroscopy (RRS).

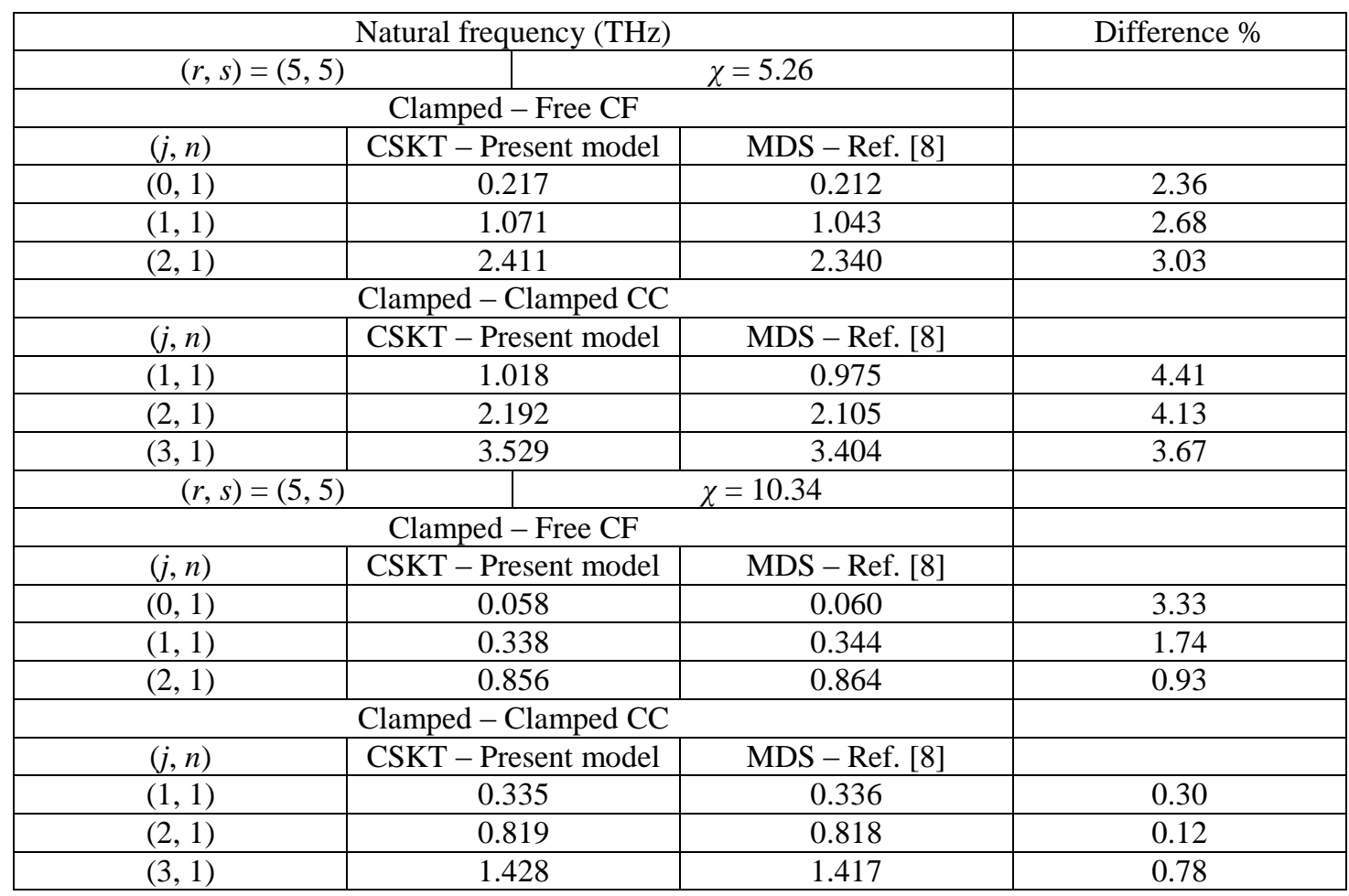

Table 3. Natural frequencies of the beam-like modes $(n=1)$ : comparisons between the complete Sanders-Koiter theory (CSKT) and the Molecular Dynamics Simulations (MDS).

\subsubsection{Beam-Like Modes (BLMs)}

The accuracy of the present numerical model is now assessed for beam-like modes $(n=1)$ by means of comparisons with MD simulations and FE analyses.

The natural frequencies of the beam-like modes obtained with the present numerical model are in good agreement with the MDS results. The comparisons shown in Table 3 for an armchair $(5,5)$ SWNT with different aspect ratios $\chi$ under clampedfree and clamped-clamped boundary conditions denote relative errors smaller than $5 \%$. 
In particular, in Table 3 it may be seen that the percent difference between the present theory and MDS, for the first four flexural modes, decreases as the length of the nanotube increases, i.e., when the influence of the boundary conditions on the natural frequencies is reduced. Furthermore, the natural frequencies under clampedfree boundary conditions are greater than those under clamped-clamped boundary conditions for the correspondent vibration modes.

\subsection{Dispersion curves of the analytical approach}

In this section, the accuracy of the analytical model based on the reduced SandersKoiter theory is studied. The equivalent parameters of Table 1 are used and numerical solutions of the transcendent equations for zigzag $(10,0)$ SWNTs of various lengths are obtained.

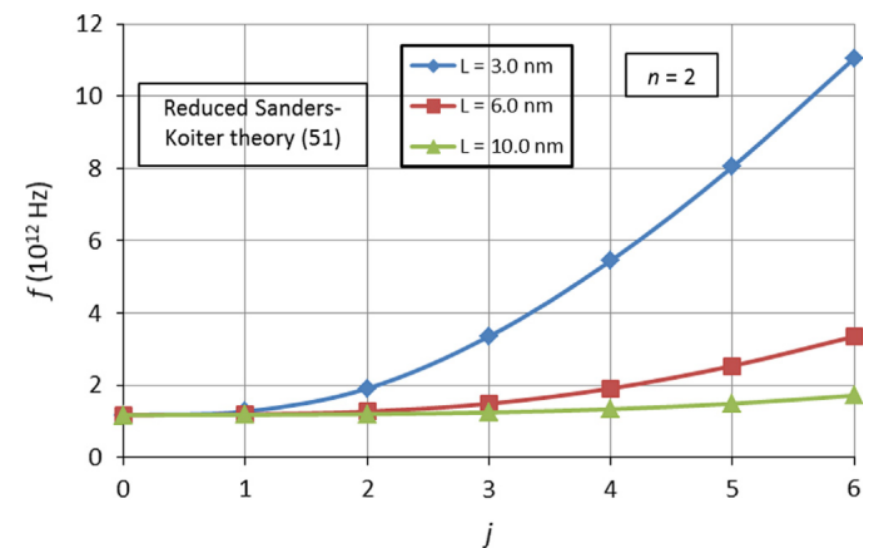

Figure 2. Eigenfrequencies of the first modes for the zigzag (10, 0) SWNT. Reduced Sanders-Koiter theory (Eq. (51)). Periodic boundary conditions. $n=2$. “--", $L=3.0 \mathrm{~nm}$; “---”, $L=6.0 \mathrm{~nm}$; “- $\triangle$-", $L=10.0 \mathrm{~nm}$

In Figure 2 the eigenfrequencies, calculated with the dispersion relation (36), are shown; different lengths are considered, the boundary conditions are periodic, $j$ is the number of half waves along the CNT axis and $n=2$ denotes the number of nodal diameters. From this figure, it is confirmed that the natural frequency of a vibrational mode $(j, n)$ increases with the number of longitudinal half waves and decreases with increasing length.

It is significant to compare the results of the dispersion relation (36), from the reduced Sanders-Koiter theory (RSKT), with those corresponding to the exact solution (Equation 1.120 of Ref. [20]). This may be seen in Figure 3. From the comparisons, it may be observed that the exact solution and the RSKT are in good agreement in the case of a small number of half waves along the CNT axis $(j \leq 3)$, when the ring and tangential shear deformation effects are small. On the other hand, the difference increases with $j$.

In Figure 4, the dependence of the eigenfrequencies of the first four modes on the ratio $(\alpha=R / L)$ of a CNT having $(r, s)=(10,0)$ is shown; the eigenfrequencies decrease as the CNT length increases. In the limit $\alpha \rightarrow 0$, the eigenfrequency is the same for any wave number $j$, because it corresponds to the eigenfrequency of a thin circular ring, in the following form [24] 


$$
\omega=\frac{n\left(n^{2}-1\right)}{\sqrt{12\left(n^{2}+1\right)}} \beta
$$

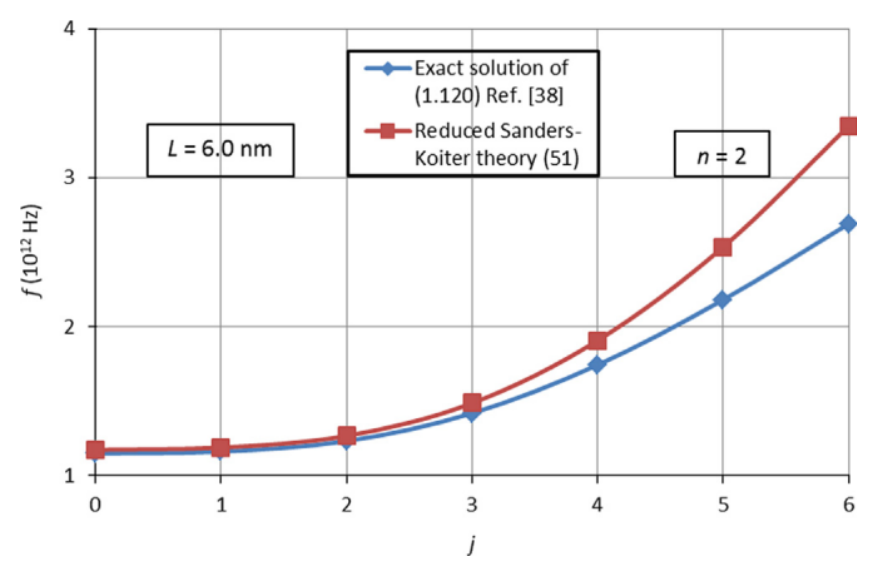

Figure 3. Dispersion curves for the zigzag $(10,0)$ SWNT. $L=6.0 \mathrm{~nm} . n=2$. “- - ", exact solution of (Eq. (1.120), Ref. [20]); “--—"”, reduced Sanders-Koiter theory (Eq. (51)).

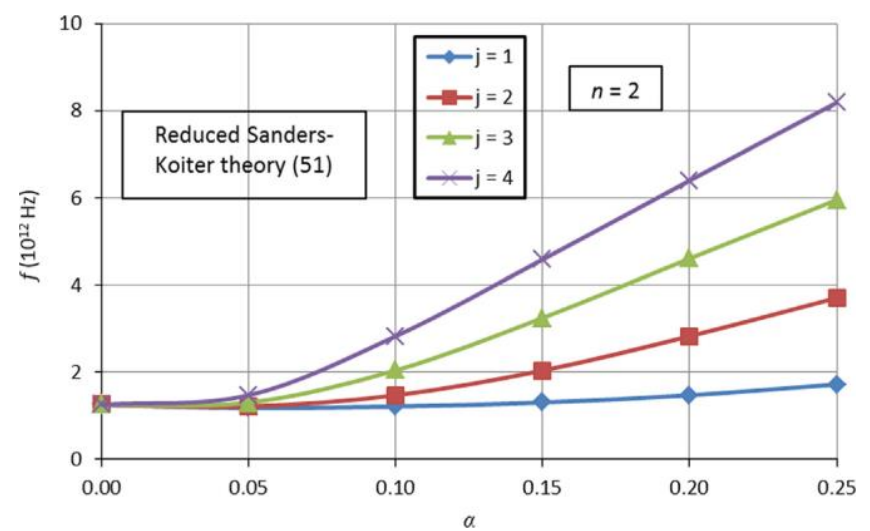

Figure 4. Dependence of the eigenfrequencies on the ratio $(\alpha=R / L)$. Zigzag $(10,0)$ SWNT. Reduced Sanders-Koiter theory (Eq. (51)). Periodic boundary conditions. $n=2$. “- -", $j=1 ;$; “--", $j$ $=2 ;$ “- $\triangle-$ ", $j=3$; “-X-”, $j=4$

\subsection{Comparison between the models}

In this section, the analytical and numerical models, based on the reduced SandersKoiter theory (RSKT) and the complete Sanders-Koiter theory (CSKT), are compared.

RSKT can be applied to the calculation of the eigenfrequencies of CNT vibrations with "angular vibration number" $n \geq 2$ [24]. The approximations of the RSKT with respect to the CSKT take into account the relative smallness of the bending and torsion stiffness with respect to those of tension and shear. This involves a correct description of long-wave modes: the range of RSKT applicability can be extended for an increased length of CNT.

The results of the RSKT and the CSKT for different lengths and boundary conditions of a $(10,0)$ SWNT are shown in Figures 5-6. From the comparisons, it can be firstly noted that the differences between RSKT and CSKT are significant for short CNTs in a large range of longitudinal waveform numbers $j$, as expected. 
Indeed, in this case the effect of the circumferential and tangential shear deformations is not negligible. Furthermore, when the length of the CNT increases, differences between RSKT and CSKT decrease, because the influence of the boundary conditions on the natural frequencies reduces.

In Figure 7, the first six mode shapes of a $(r=10, s=0, \chi=12.8)$ SWNT with free edges for the circumferential wavenumber $(n=2)$ are reported. These mode shapes are obtained by using the equivalent parameters $(h, E, v, \rho)$ of Table 1 . The modes $(j$ $=0, n=2)$ and $(j=1, n=2)$ correspond to the Rayleigh's inextensional symmetric mode (uniform vibration) and Love's asymmetric mode (one-half of the wave length), respectively (Ref. [20]). The modes $(j=2, n=2)$ (wave length) and $(j=4, n$ $=2)$ are symmetric, the modes $(j=3, n=2)$ and $(j=5, n=2)$ are asymmetric, with respect to the central transversal section of the CNT. Such graphical representation of modes could be useful for interpreting the previous results and comparisons.
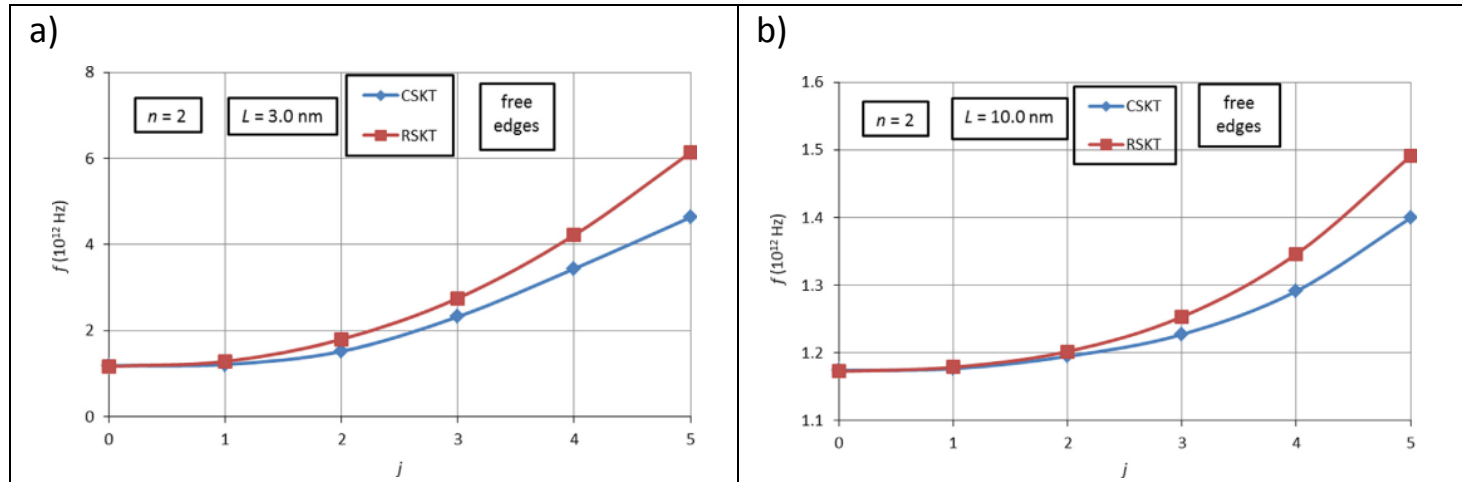

Figure 5. Natural frequencies, zigzag $(10,0)$ SWNT. $n=2$. Free edges. " $\$$-", complete SandersKoiter theory (CSKT); “--"”, reduced Sanders-Koiter theory (RSKT). a) $L=3.0 \mathrm{~nm}$; b) $L=10.0 \mathrm{~nm}$.
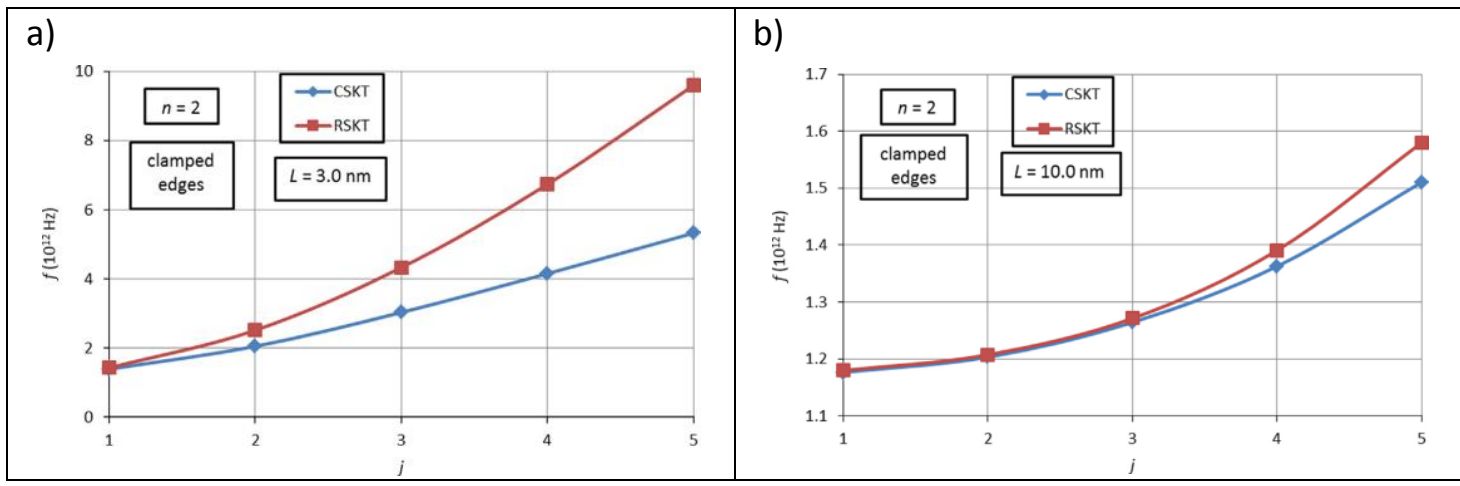

Figure 7. Natural frequencies, zigzag $(10,0)$ SWNT. $n=2$. Clamped edges. “- $-”$, complete SandersKoiter theory (CSKT); “- "-", reduced Sanders-Koiter theory (RSKT). a) $L=3.0 \mathrm{~nm}$; b) $L=10.0 \mathrm{~nm}$. 


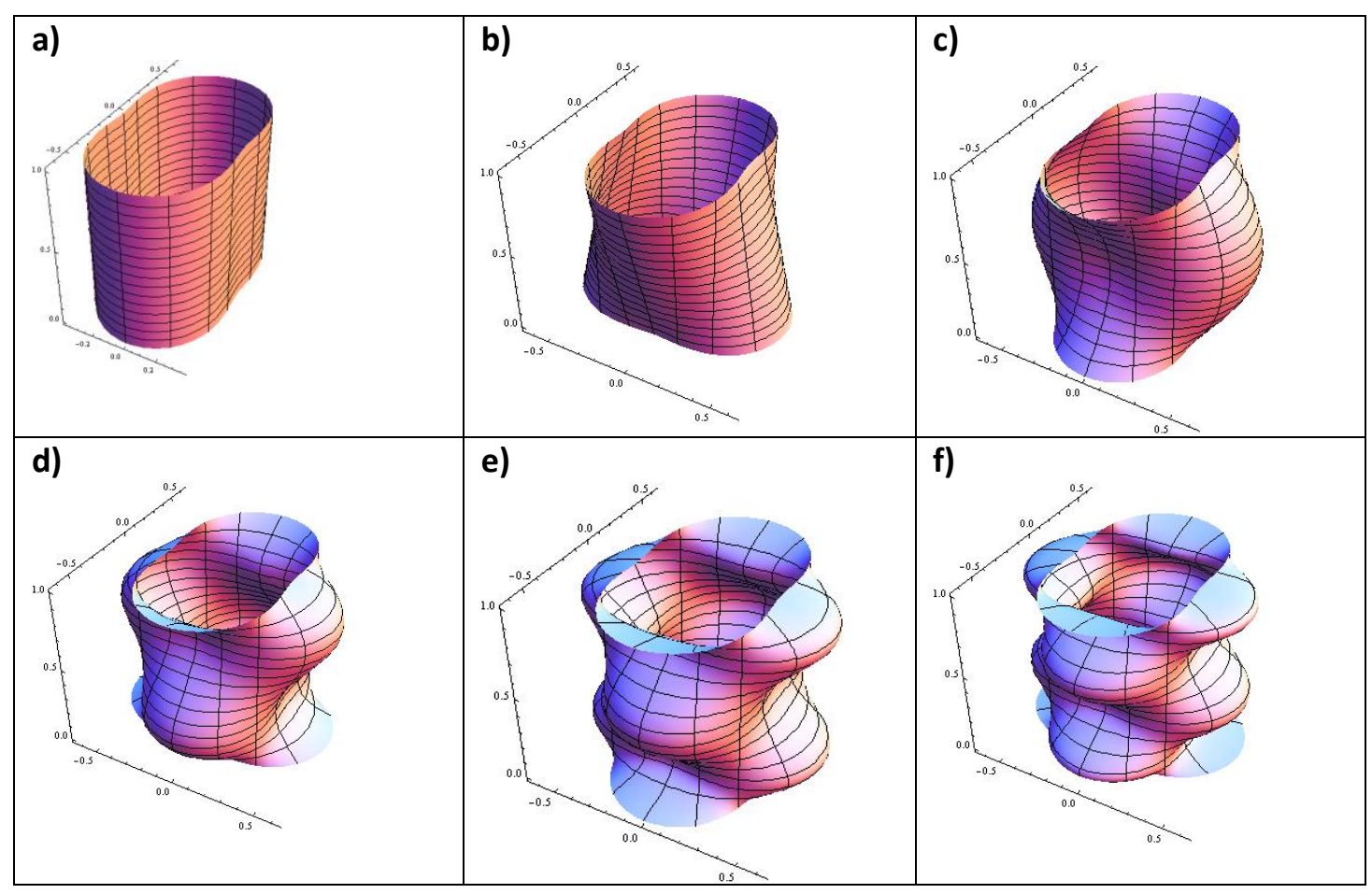

Figure 7. Mode shapes of the SWNT $(r=10, s=0, L=10.0 \mathrm{~nm})$; equivalent parameters of Table 1; free edges. a) $(j=0, n=2)$. b) $(j=1, n=2)$. c) $(j=2, n=2)$. d) $(j=3, n=2)$. e) $(j=4, n=2)$. f) $(j=$ $5, n=2)$.

\section{Conclusions}

In this paper, a comprehensive analysis of the vibration spectrum of Single-Walled Carbon Nanotubes in the framework of the continuum shell theory is presented.

Size effects are neglected because the present paper is focused on low frequencies of SWNTs with large aspect ratios and diameters.

Significant results concerning applicability, experimental verification and possibility of analytical study for the most important boundary conditions are presented.

1. The results obtained from a numerical model based on the Sanders-Koiter shell theory, which does not introduce any restriction on the geometric parameters and wave numbers, are in excellent agreement with the existing experimental and molecular dynamics data.

$\rightarrow$ a. The theory is accurate for SWNTs having large aspect ratios and diameters.

2. An analytical model based on a reduced version of the Sanders-Koiter shell theory is presented. It supplies a new tool valid in a wide parameter range, where other simpler elastic shell theories (e.g., Donnell shallow shell or semi-momentless theories) are not accurate.

$\rightarrow$ a. The analytical model gives results close to those from the numerical model for low-frequency normal vibrations.

$\rightarrow$ b. The possibility of having an efficient analytical solution allows the spatial structure of normal modes to be clarified: in particular, it reveals the edge effects and their prolongation, which are dependent on the geometric parameters and wave numbers. 
Starting from the two models presented here, a future work will consider the energy distribution on the carbon nanotube in nonlinear field, including the presence of conjugate modes, which can give rise to travelling-wave response moving along the circumferential direction of the CNT.

\section{References}

[1] Iijima S. Helical microtubules of graphitic carbon. Nature 354 (1991) 56-58.

[2] Rao AM, Richter E, Bandow S, Chase B, Eklund PC, Williams KA, Fang S, Subbaswamy KR, Menon M, Thess A, Smalley RE, Dresselhaus G, Dresselhaus MS. Diameter-Selective Raman Scattering from Vibrational Modes in Carbon Nanotubes. Science 275 (1997) 187-191.

[3] Bandow S, Asaka S, Saito Y, Rao AM, Grigorian L, Richter E, Eklund PC. Effect of the Growth Temperature on the Diameter Distribution and Chirality of Single-Wall Carbon Nanotubes. Physical Review Letters 80(17) (1998) 3779-3782.

[4] Jorio A, Saito R, Hafner JH, Lieber CM, Hunter M, McClure T, Dresselhaus G, Dresselhaus MS. Structural $(n, m)$ Determination of Isolated Single-Wall Carbon Nanotubes by Resonant Raman Scattering. Physical Review Letters 86(6) (2001) 1118-1121.

[5] Gupta SS, Bosco FG, Batra RC. Breakdown of structural models for vibrations of single-wall zigzag carbon nanotubes. Journal of Applied Physics 106 (2009) 063527(9).

[6] Cheng HC, Liu YL, Wu CH, Chen WH. On radial breathing vibration of carbon nanotubes. Computational Methods in Applied Mechanical Engineering 199 (2010) 2820-2827.

[7] Gupta SS, Bosco FG, Batra RC. Wall thickness and elastic moduli of singlewalled carbon nanotubes from frequencies of axial, torsional and inextensional modes of vibration. Computational Materials Science 47 (2010) 1049-1059.

[8] Duan WH, Wang CM, Zhang YY. Calibration of nonlocal scaling effect parameter for free vibration of carbon nanotubes by molecular dynamics. Journal of Applied Physics 101 (2007) 024305(7).

[9] Sakhaee-Pour A, Ahmadian MT, Vafai A. Vibration analysis of single-walled carbon nanotubes using beam element. Thin-Walled Structures 47 (2009) 646-652.

[10] Arghavan S, Singh AV. On the vibrations of single-walled carbon nanotubes. Journal of Sound and Vibration 330 (2011) 3102-3122.

[11] Odegard GM, Gates TS, Nicholson LM, Wise KE. Equivalent-Continuum Modeling of Nano-Structured Materials. Composites Science and Technology 62(14) (2002) 1869-1880.

[12] Lu P, Lee HP, Lu C, Zhang PQ. Application of nonlocal beam models for carbon nanotubes. International Journal of Solids and Structures 44 (2007) 52895300 .

[13] Yang S, Yu S, Kyoung W, Han DS, Cho M. Multiscale modeling of sizedependent elastic properties of carbon nanotube/polymer nanocomposites with interfacial imperfections. Polymer 53 (2012) 623-633. 
[14] Kiani K. A meshless approach for free transverse vibration of embedded singlewalled nanotubes with arbitrary boundary conditions accounting for nonlocal effect. International Journal of Mechanical Sciences 52(10) (2010) 1343-1356.

[15] Wang L, Hu H. Flexural wave propagation in single-walled carbon nanotubes. Physical Review B 71 (2005) 195412(7).

[16] He XQ, Eisenberger M, Liew KM. The effect of Van der Waals interaction modeling on the vibration characteristics of multiwalled carbon nanotubes. Journal of Applied Physics 100 (2006) 124317(12).

[17] Peng J, Wu J, Hwang KC, Song J, Huang Y. Can a Single-Wall Carbon Nanotube Be Modeled as a Thin Shell? Journal of the Mechanics and Physics of Solids 56 (2008) 2213-2224.

[18] Yakobson BI, Brabec CJ, Bernholc J. Nanomechanics of Carbon Tubes: Instabilities beyond Linear Response. Physical Review Letters 76(14) (1996) 25112514.

[19] Wang CY, Ru CQ, Mioduchowski A. Applicability and Limitations of Simplified Elastic Shell Equations for Carbon Nanotubes. Journal of Applied Mechanics 71 (2004) 622-631.

[20] Leissa AW. Vibrations of Shells. Government Printing Office, Washington DC, 1973.

[21] Pellicano F. Vibrations of circular cylindrical shells: Theory and experiments. J2urnal of Sound and Vibration 303 (2007) 154-170.

[22] Pellicano F. Dynamic instability of a circular cylindrical shell carrying a top mass under base excitation: Experiments and theory. International Journal of Solids and Structures 48 (2011)

408-427.

[23] Strozzi M, Pellicano F. Nonlinear vibrations of functionally graded cylindrical shells. Thin-Walled Structures 67 (2013) 63-77.

[24] Smirnov VV, Shepelev DS, Manevitch LI. Localization of bending vibrations in the Single-Walled Carbon Nanotubes. Nanosystems: Physics, Chemistry, Mathematics 2(2) (2011) 102-106. 